\title{
Atrial Metastatic Tumor: Transthoracic and Transesophageal Echocardiograph Imaging
}

\author{
Konstantinos Lampropoulos ${ }^{\mathrm{a}, \mathrm{c}}$, Dimitrios Kotsas ${ }^{\mathrm{a}}$, Christina Parmaki ${ }^{\mathrm{a}}$, Themistoklis Iliopoulos ${ }^{\mathrm{b}}$
}

\begin{abstract}
A 68-year-old male patient was admitted to our hospital because of weakness, dyspnea on exertion and palpitations. Transthoracic and transesophageal echocardiograph revealed a dilated left atrium with an immobile intracavitary mass, without signs of left atrium outflow obstruction. Bronchoscopy was performed and the histopathologic review of the samples obtained was consistent with a primary lung squamous cell carcinoma.
\end{abstract}

Keywords: Atrial metastatic tumor; Transthoracic; Transesophageal; Echocardiography

\section{Introduction}

Metastases to the heart and pericardium are much more common than primary cardiac tumors and are generally associated with a poor prognosis [1-3]. Metastases to the heart were found in $1.23 \%$ of 12,485 consecutive autopsies, compared with a $0.056 \%$ prevalence of primary cardiac tumors [1]. In autopsies where a malignant neoplasm was identified, cardiac metastases were found in $9.7 \%-10.7 \%$ of cases [4-7]. Noncardiac tumors may invade the heart and pericardium by means of lymphatic or hematogenous dissemination, local extension, or transvenously. Tumors that are most likely to involve the heart and pericardium include cancers of the lung and breast, melanoma, and lymphoma [5, 6, 8]. These tumors may involve the heart and pericardium by one of four

Manuscript accepted for publication July 5, 2013

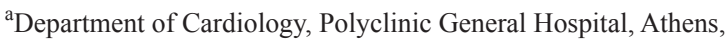
Greece

${ }^{\mathrm{b}}$ Department of Cardiology, 251 General Air Force Hospital, Athens, Greece

${ }^{\mathrm{c} C}$ Corresponding Author: Konstantinos Lampropoulos, 31, L.Porfyra St., Athens 16673, Greece. Email: konlampropoulos@yahoo.gr

doi: http://dx.doi.org/10.4021/jmc1390w pathways: retrograde lymphatic extension, hematogenous spread, direct contiguous extension, or transvenous extension [9].

Whereas two- and three-dimensional (2D, 3D) echocardiography is the method of imaging most frequently used to examine the heart and pericardium noninvasively, magnetic resonance (MR) imaging and computed tomography (CT) offer advantages when metastatic disease is in question. Both imaging modalities provide a large examining field which allows evaluation of disease throughout the thorax [10].

We report the case of a patient with a primary lung squamous cell carcinoma with cardiac metastatic disease, diagnosed with chest X-ray and cardiac ultrasonography.

\section{Case Report}

In this report we present a 68 -year-old male patient who was admitted to our hospital because of weakness, dyspnea on exertion and palpitations. He was an active smoker and complained of weight loss of $25 \mathrm{lbs}$ over the last three months. Physical examination and blood tests at the Emergency Department were unremarkable. However, his electrocardiogram showed atrial fibrillation and the chest X-ray revealed a right lower lung field shadow. A transthoracic echocardiogram was arranged and a large tumor was pictured in the left atrium $(39.7 \times 36.4 \mathrm{~mm}$ diameter $)$, attached to its posterior wall (Fig. 1A, B). Transesophageal echocardiograph confirmed a dilated left atrium with an immobile mass in the cavity, without any signs of left atrial outflow obstruction (Fig. 1C, D). The patient was further evaluated with thoracic CT scanning that confirmed the presence of a right lung mass. Subsequently, a bronchoscopic study with tumor sampling was performed and there was histopathologic confirmation of a primary lung squamous cell carcinoma.

\section{Discussion}

Metastatic involvement of the heart and pericardium may go unrecognized until autopsy. Impairment of cardiac function occurs in approximately $30 \%$ of patients and is usually at- 


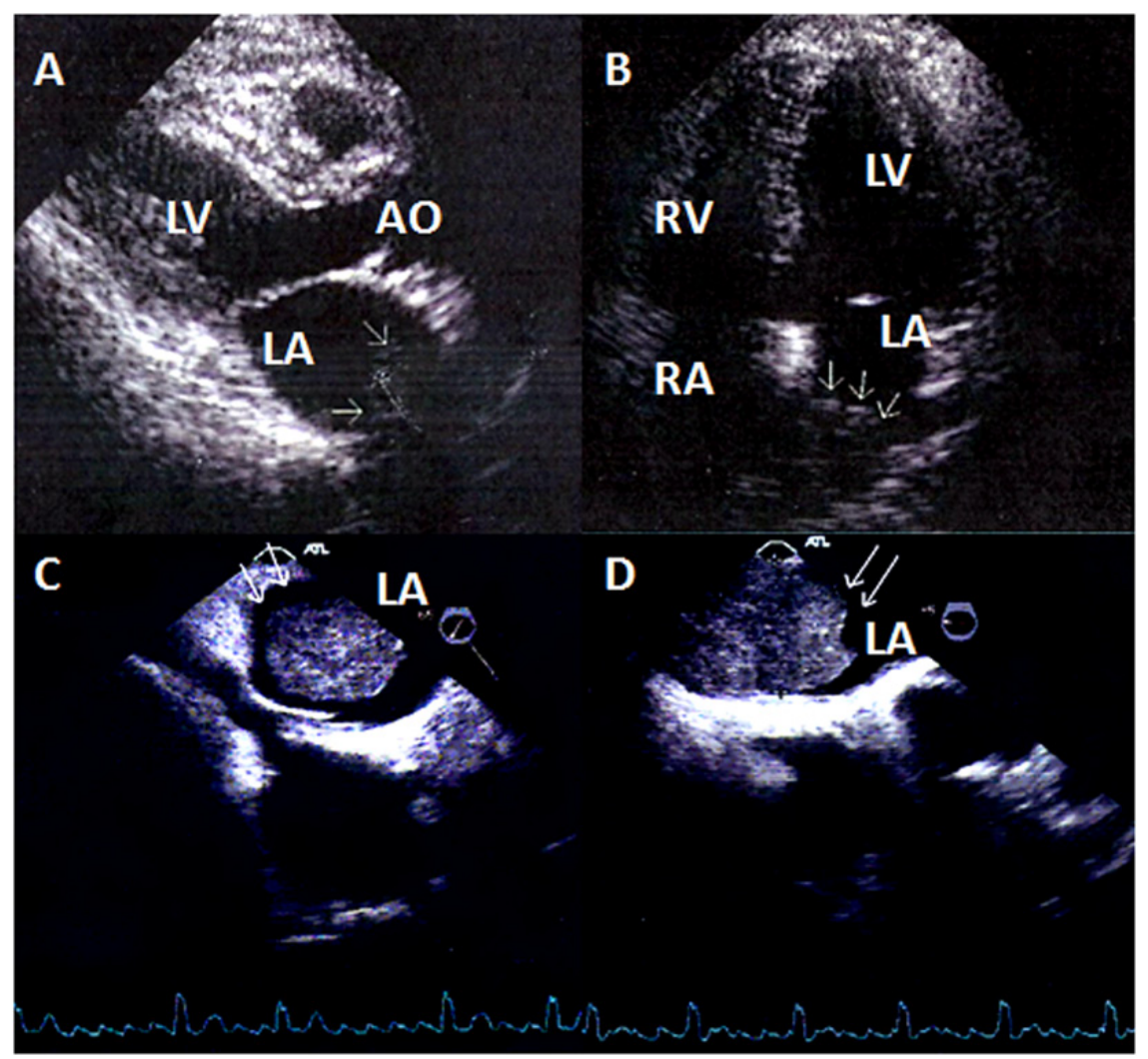

Figure 1. (A) Two-dimensional echocardiogram, parasternal long axis view of the left ventricle. In the left atrium arrows show the metastatic tumor. (B) Two-dimensional echocardiogram, four-chamber apical view. In the left atrium the arrows show the metastatic tumor. (C) Transesophageal echocardiogram $\left(+65^{\circ}\right)$. The metastatic tumor in the left atrium. (D) Transesophageal echocardiogram $\left(-5^{\circ}\right)$. The metastatic tumor in the left atrium.

tributable to pericardial effusion [11]. In literature a highly variable incidence of cardiac metastases is reported, ranging from $2.3 \%$ and $18.3 \%[8,12]$. Shortness of breath is prominent in the clinical presentation, often out of proportion to the radiographic findings, and may be attributed to a pericardial effusion or an associated pleural effusion [13, 14]. Presenting complaints also include cough, anterior thoracic pain, pleuritic chest pain, or peripheral edema $[15,16]$. Arrhythmias in patients with cardiac metastases is usually the result of concomitant factors such as hypoxemia, electrolyte imbalance, or anemia; however, arrhythmia can be secondary to tumors involving autonomic fibers or encroaching on the coronary arteries [17]. Overall, arrhythmia is the most prevalent manifestation of myocardial involvement by metastatic tumors $[12,18]$. The occurrence of arrhythmia without an obvious trigger in a patient with known malignancy suggests the possibility of metastatic myocardial involvement. The type of arrhythmia depends on the size of the tumor and its location relative to the conduction system of the heart. In our patient, palpitation revealed persistent atrial fibrillation, while transthoracic, transesophageal echocardiograph, CT scanning and bronchoscopic study confirmed the diagnosis of a primary lung squamous cell carcinoma.
Lung carcinoma is postulated to metastasize to the heart most often by direct invasion, followed by lymphatic and hematogenous spread. The right side of the heart is more frequently involved by metastatic lesions than the left side. In this case, the most likely culprit is hematogenous spread as cancer cells had direct access through the right lower pulmonary vein to the left atrium.

Differential diagnosis of intracavitary mass lesions includes benign and malignant primary cardiac tumors like myxoma, and various other space occupying lesions in the form of thrombus, vegetations and foreign bodies. Infectious and non-bacterial thrombotic or marantic endocarditis can also present with similar findings. It is important for admitting physicians to bear in mind that intramural metastases should be considered in the differential diagnosis of myocardial infarction and pericardial metastases in the differential diagnosis of pericardial effusion.

Imaging studies valuable to establish the diagnosis include (2D, 3D) transthoracic and transesophageal echocardiogram [19], CT scan, MR imaging [10] and angiography. Still, metastatic cardiac lesions, most of which are clinically silent, are often only found post-mortem. Other possible complications include peripheral embolism and sudden out- 
flow tract obstruction. Sadly, the prognosis is poor if the patient is unresponsive to chemotherapy.

\section{Conclusions}

Real time 2D and 3D transthoraric echocardiography is a low cost, readily accessible, reproducible, non ionizing radiation and non-invasive technique that provides an indispensable tool for the diagnosis of cardiac tumors. In addition, 2D and 3D transesophageal echocardiography can frequently provide essential supplementary information, obviating the need for further expensive and time consuming imaging tests.

\section{Conflict of Interest}

There was no financial relationship between the authors and the subject matter.

\section{References}

1. Lam KY, Dickens P, Chan AC. Tumors of the heart. A 20-year experience with a review of 12,485 consecutive autopsies. Arch Pathol Lab Med. 1993;117(10):10271031.

2. Luk A, Ahn E, Vaideeswar P, Butany JW. Pericardial tumors. Semin Diagn Pathol. 2008;25(1):47-53.

3. Mukai K, Shinkai T, Tominaga K, Shimosato Y. The incidence of secondary tumors of the heart and pericardium: a 10-year study. Jpn J Clin Oncol. 1988;18(3):195201.

4. Nakayama R, Yoneyama T, Takatani O, Kimura K. A study of metastatic tumors to the heart, pericardium and great vessels. I. Incidences of metastases to the heart, pericardium and great vessels. Jpn Heart J. 1966;7(3):227-234.

5. Abraham KP, Reddy V, Gattuso P. Neoplasms metastatic to the heart: review of 3314 consecutive autopsies. Am J Cardiovasc Pathol. 1990;3(3):195-198.

6. Klatt EC, Heitz DR. Cardiac metastases. Cancer.
1990;65(6):1456-1459.

7. Amano J, Nakayama J, Yoshimura Y, Ikeda U. Clinical classification of cardiovascular tumors and tumor-like lesions, and its incidences. Gen Thorac Cardiovasc Surg. 2013;61(8):435-447.

8. Sarjeant JM, Butany J, Cusimano RJ. Cancer of the heart: epidemiology and management of primary tumors and metastases. Am J Cardiovasc Drugs. 2003;3(6):407421.

9. Schoen FJ, Berger BM, Guerina NG. Cardiac effects of noncardiac neoplasms. Cardiol Clin. 1984;2(4):657670.

10. Chiles C, Woodard PK, Gutierrez FR, Link KM. Metastatic involvement of the heart and pericardium: CT and MR imaging. Radiographics. 2001;21(2):439-449.

11. Thurber DL, Edwards JE, Achor RW. Secondary malignant tumors of the pericardium. Circulation. 1962;26:228-241.

12. Debourdeau P, Gligorov J, Teixeira L, Aletti M, Zammit C. [Malignant cardiac tumors]. Bull Cancer. 2004;91(Suppl 3):136-146.

13. Quraishi MA, Costanzi JJ, Hokanson J. The natural history of lung cancer with pericardial metastases. Cancer. 1983;51(4):740-742.

14. Kisselbach C, Ristic AD, Pankuweit S, Karatolius K, Maisch B. [Women and pericardial neoplastic manifestations of the heart and pericardium]. Herz. 2005;30(5):409-415; quiz 429-430.

15. Bussani R, De-Giorgio F, Abbate A, Silvestri F. Cardiac metastases. J Clin Pathol. 2007;60(1):27-34.

16. Lamba G, Frishman WH. Cardiac and pericardial tumors. Cardiol Rev. 2012;20(5):237-252.

17. Reynen K, Kockeritz U, Strasser RH. Metastases to the heart. Ann Oncol. 2004;15(3):375-381.

18. Waller BF, Gering LE, Branyas NA, Slack JD. Anatomy, histology, and pathology of the cardiac conduction system--Part IV. Clin Cardiol. 1993;16(6):507-511.

19. Lampropoulos K, Bogaert J, Voigt JU, Budts W. Left atrial myxoma. Evaluation with transoesophageal echocardiographic and real time three-dimensional imaging. Acta Clin Belg. 2011;66(4):318-320. 